\title{
Thermal gravity-driven convection of near-critical helium in enclosures
}

\author{
V.I. Polezhaev and E.B. Soboleva \\ Institute for Problems in Mechanics RAS, pr. Vernadskogo, 101, b.1, Moscow, 119526, Russia \\ E-mail: soboleva@ipmnet.ru
}

Received December 19, 2002

\begin{abstract}
The results of numerical simulation of Rayleigh - Benard convection in ${ }^{3} \mathrm{He}$ near the thermodynamic critical point are presented. The mathematical model including the full Navier-Stokes equations with two-scale splitting of the pressure and the van der Waals equation of state is applied. The known experimental data on ${ }^{3} \mathrm{He}$ are used in simulations. On the basis of the calibration laws the «real» Rayleigh and Prandtl numbers are estimated. It is shown that one should agree these «real» criteria of similarity in the model and physical media to approach results of simulations to experiments. The Rayleigh number characterising the convection onset is defined from obtained numerical data. This number is shown to be in a good agreement with known experimental and theoretical values.
\end{abstract}

PACS: 05.70.Ln

\section{Introduction}

In the vicinity of the thermodynamic critical point media named as near-critical fluids display specific thermodynamic and kinetic properties [1,2]. They posses an asymptotic discrepancy of the specific heat at constant pressure, coefficient of isothermal compressibility, and thermal expansion coefficient. In the other hand, they have the heat diffusion coefficient tending to zero. These abnormal properties lead to specific heat transfer and interesting hydrodynamic effects as compared with a perfect gas.

We will simulate numerically thermal gravity-driven convection on the basis of the hydrodynamic consideration with the van der Waals equation of state. The classical problem of steady-state Rayleigh-Benard convection (with bottom heating) well-known for a perfect gas [3] will be solved for a near-critical fluid and will be compared with experimental data. Parameters of numerical simulation will be estimated on the basis of the physical properties of helium and the approach of the model fluid to the real one will be fulfilled. The Rayleigh-Benard convection was simulated in intensive near-critical flows [4], but we will investigate the onset of convection.

\section{Mathematical model of a non-perfect gas}

The full Navier - Stokes equations and the equation of energy for a non-perfect gas with the van der Waals equation of state are applied. Two-scale splitting of the pressure into volume-average and dynamic parts is used [5]. To close the set of equations the integral mass balance is involved. The governing equations in dimensionless form are as follows [6]:

$$
\begin{gathered}
\frac{\partial \rho}{\partial t}+\nabla(\rho \mathbf{U})=0 \\
\rho \frac{\partial U}{\partial t}+\rho(\mathbf{U} \nabla) \mathbf{U}=-\nabla p+\frac{1}{\operatorname{Re}(2 \nabla(\eta \dot{D})-} \\
\left.-\nabla\left(\frac{2}{3} \eta-\zeta\right) \nabla \mathbf{U}\right)+\frac{\operatorname{Ra}}{\operatorname{Pr} \Theta \operatorname{Re}^{2}} \rho \mathbf{g}, \\
\rho \frac{\partial T}{\partial t}+\rho(\mathbf{U} \nabla) T=-\left(\gamma_{0}-1\right) T\left(\frac{\partial P}{\partial T}\right)_{\rho} \nabla \mathbf{U}+ \\
+\frac{\gamma_{0}}{\operatorname{Re} \operatorname{Pr}} \nabla(\lambda \nabla T)+\frac{\gamma_{0}\left(\gamma_{0}-1\right) M^{2}}{\operatorname{Re}} \times \\
\times\left(2 \eta \dot{D}^{2}-\nabla\left(\frac{2}{3} \eta-\zeta\right)(\nabla \mathbf{U})^{2}\right),
\end{gathered}
$$




$$
\begin{gathered}
P=\rho T /(1-b \rho)-a \rho^{2}, a=9 / 8, b=1 / 3, \\
P=\langle P\rangle+\gamma_{0} M^{2} p, \\
\int_{V} p d v=0 .
\end{gathered}
$$

Here $\rho, \mathbf{U}, \dot{D}$, and $T$ are the density, the velocity, the strain rate tensor, and the temperature; $P,\langle P\rangle$, and $p$ are the total pressure, the volume-average, and dynamic pressure components; $\mathbf{g}$ is the mass force acceleration; $\eta, \zeta$, and $\lambda$ are the coefficients of dynamic, bulk viscosity, and heat conductivity; $d v$ is an elemental volume, $V$ is the overall volume. Dimensionless values are without primes. The characteristic scales are: length $l^{\prime}$, velocity $U^{\prime}$, time $l^{\prime} / U^{\prime}$, strain rate $U^{\prime} / l^{\prime}$, the Earth's gravity force acceleration $g^{\prime}$, the critical values $\rho_{c}^{\prime}, T_{c}^{\prime}$ and the values $\lambda_{0}^{\prime}, \eta_{0}^{\prime}, c_{v 0}^{\prime}$ corresponding to a perfect gas; $B^{\prime}=R^{\prime} / \mu_{g}^{\prime}, R^{\prime}$ is the perfect gas constant, $\mu_{g}^{\prime}$ is the molar mass. Primes denote the dimensional values, subscript «c» - the critical values, subscript $\ll 0 »-$ a perfect gas.

The dimensionless parameters (the ratio of specific heats, characteristic temperature difference, the Reynolds, Mach, Prandtl, Rayleigh numbers for a perfect gas) are

$$
\begin{aligned}
& \gamma_{0}=1+\frac{B^{\prime}}{c_{v 0}^{\prime}}, \quad \Theta=\frac{\Theta^{\prime}}{T_{c}^{\prime}}, \quad \operatorname{Re}=\frac{\rho_{c}^{\prime} U^{\prime} l^{\prime}}{\eta_{0}^{\prime}}, \quad \mathrm{M}=\frac{U^{\prime}}{\sqrt{\gamma_{0} B^{\prime} T_{c}^{\prime}}}, \\
& \mathrm{Ra}=\frac{\Theta^{\prime} g^{\prime} l^{3} \rho_{c}^{\prime 2}\left(c_{v 0}^{\prime}+B^{\prime}\right)}{T_{c}^{\prime} \lambda_{0}^{\prime} \eta_{0}^{\prime}}, \quad \operatorname{Pr}=\frac{\left(c_{v 0}^{\prime}+B^{\prime}\right) \eta_{0}^{\prime}}{\lambda_{0}^{\prime}} .
\end{aligned}
$$

The total pressure $P$ is decomposed into two parts (the volume-average $\langle P\rangle$ and dynamic $p$ components) and the parts are normalized using the different scales $\left(B^{\prime} \rho_{c}^{\prime} T_{c}^{\prime}-\right.$ for $\langle P\rangle$ and $\rho_{c}^{\prime} U^{\prime 2}-$ for $\left.p\right)$. Splitting and two-scale representation of the pressure are applied for successful simulating both acoustic and low-speed flows with the rather large time steep.

Considering conditions close to the critical isochore the near-critical features are associated with the temperature distance from the critical point (or reduced temperature) $\varepsilon=\left(T^{\prime}-T_{c}^{\prime}\right) / T_{c}^{\prime}$. The coefficient $\lambda$ increases as $\varepsilon \rightarrow 0$ and may be fitted to the relation [1]

$$
\lambda=1+\Lambda \varepsilon^{-\psi},
$$

the coefficient $\eta$ is supposed to be constant, $\zeta$ is equal to zero.

The convective motion is characterized by the Rayleigh and Prandtl numbers. With approach to the critical point these criteria of similarity tend to infinity, while the values of $\mathrm{Ra}$ and $\mathrm{Pr}$ in the governing equations based on the perfect-gas parameters remain the same. To describe near-critical convection completely we consider the «real» Rayleigh $\mathrm{Ra}_{r}$ and the «real» Prandtl $\operatorname{Pr}_{r}$ numbers which take the real physical properties in the vicinity of the critical point into account. They are signed by subscript «r» and expressed by the calibration laws (near critical isochore) [7]

$$
\begin{gathered}
\operatorname{Ra}_{r}=\frac{2}{3} \varepsilon^{-1}\left(\frac{1}{\gamma_{0}}+\frac{\gamma_{0}-1}{\gamma_{0}} \frac{1+\varepsilon}{\varepsilon}\right) \frac{1}{\lambda} \mathrm{Ra}, \\
\operatorname{Pr}_{r}=\left(\frac{1}{\gamma_{0}}+\frac{\gamma_{0}-1}{\gamma_{0}} \frac{1+\varepsilon}{\varepsilon}\right) \frac{1}{\lambda} \operatorname{Pr} .
\end{gathered}
$$

The values $\operatorname{Ra}_{r}$ and $\operatorname{Pr}_{r}$ diverging asymptotically with approach to the critical point:

$$
\begin{gathered}
\operatorname{Ra}_{r} / \operatorname{Ra} \sim \varepsilon^{\psi-2} \rightarrow \infty, \operatorname{Pr}_{r} / \operatorname{Pr} \sim \varepsilon^{\psi-1} \rightarrow \infty \quad(\psi<1) \\
\text { as } \varepsilon \rightarrow 0
\end{gathered}
$$

\section{Physical properties of helium and parameters of Rayleigh-Benard convection near the critical point}

The critical point in ${ }^{3} \mathrm{He}$ is characterized by parameters $[8,9] \quad T_{c}^{\prime}=3.3189 \mathrm{~K}, \rho_{c}^{\prime}=0.0414 \mathrm{~g} / \mathrm{cm}^{3}$, $P_{c}^{\prime}=0,117 \mathrm{MPa}$. We will consider Rayleigh - Benard convection in ${ }^{3} \mathrm{He}$ studied experimentally $[8,9]$ and will use the physical properties of ${ }^{3} \mathrm{He}$ at reduced temperature $5 \cdot 10^{-4} \leq \varepsilon \leq 0.2[10]$. To choose model parameters we need to treat available data.

We take data on the thermal conductivity $\lambda^{\prime}$ and fit them to the relation (8) considering the dimensionless magnitude $\lambda=\lambda^{\prime} / \lambda_{0}^{\prime}$, where $\lambda_{0}^{\prime}$ is the scale value. The constants $\Lambda, \psi$ and $\lambda_{0}^{\prime}$ are defined giving the optimal curve

$$
\lambda=1+0.0149 \varepsilon^{-0.645}
$$

normalized by the scale $\lambda_{0}^{\prime}=1.73 \cdot 10^{-4} \mathrm{~W} /(\mathrm{cm} \cdot \mathrm{K})$. The curve (10) in compare with experimental points [10] is plotted in Fig. 1 showing a good agreement between them.

The data of experiments [10] allow to find the specific heat at constant pressure $c_{p}$ and the thermal expansion coefficient $\beta$ according to the relations in dimensionless form

$$
c_{p}=c_{\vartheta} \gamma, \beta=c_{v}(\gamma-1) /\left[(1+\varepsilon)(\partial P / \partial T)_{\rho}\right] .
$$

Then one can evaluate the experimental Rayleigh number including the real physical properties as

$$
\operatorname{Ra}_{r}=\operatorname{Ra} \beta c_{p} Z /\left(\lambda \gamma_{0}\right)
$$

Here, $Z=P_{c}^{\prime} /\left(B^{\prime} \rho_{c}^{\prime} T_{c}^{\prime}\right)$ is the compressibility factor. The classical value of the specific heats for a perfect gas $\gamma_{0}=1.67$ is used. The magnitude of $\mathrm{Ra}$ is estimated on 


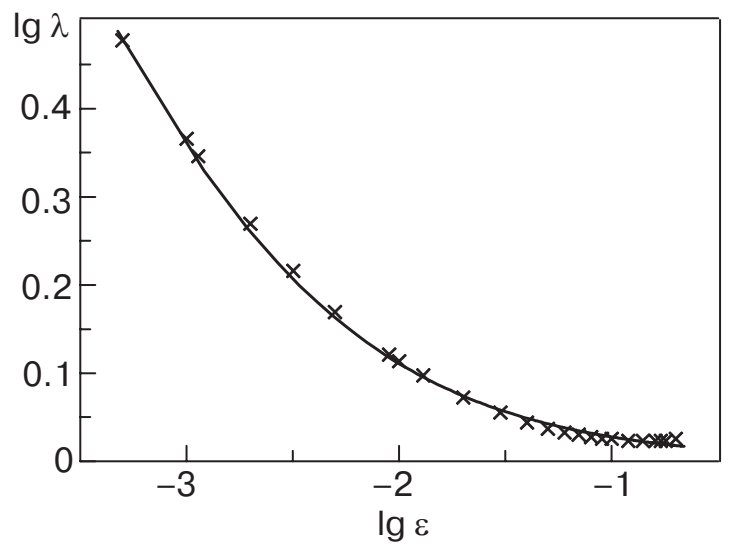

Fig. 1. Calculated fitting curve of the thermal conductivity $\lambda$ (10) (solid line) and experimental points (marks) [10] as functions of reduced temperature $\varepsilon$ in logarithmic coordinates.

the basis of the experiments $[8,9]$ and properties of helium far from the critical point. We take the typical temperature difference $\Theta^{\prime}$ from the experimental range and assume for the sake of definiteness that it is $10 \mu \mathrm{K}$. We have $l^{\prime}=0,1 \mathrm{~cm}, g_{0}^{\prime}=9,8 \cdot 10^{2} \mathrm{~cm} / \mathrm{s}^{2}, \Theta^{\prime}=10^{-5} \mathrm{~K}$, $\eta_{0}^{\prime}=16.7 \cdot 10^{-6} \mathrm{~g} /(\mathrm{cm} \cdot \mathrm{s}), \lambda_{0}^{\prime}=1.73 \cdot 10^{-4} \mathrm{~W} /(\mathrm{cm} \cdot \mathrm{K})$, $c_{v 0}^{\prime}=3.12 \cdot 10^{7} \mathrm{erg} /(\mathrm{K} \cdot \mathrm{g})$. The experimental Prandtl numbers $\operatorname{Pr}_{r}$ are taken from [10] and they allow to obtain Pr according to (9). Consequently, the values appropriate to the physical properties are $\mathrm{Ra}=9.10$, $\operatorname{Pr}=0.501$.

The obtained results show some discrepancies between experimental and model criteria of similarity which increase with decreasing in $\varepsilon$. The reason of these discrepancies is believed to be due to the equation of state. The van der Waals form and the other analytic equations of this type, for example, Redlich-Kwong one applied in [11], give the fixed critical exponents different from the experimental values. The scaled equations of state supported by the renormalization-group theory $[12,13]$ are known to ensure the best agreement between the theoretical and experimental exponents, but they are applicable only asymptotically when $\varepsilon<<1$. We cannot employ any scaled equation because of a wide range of $\varepsilon$ in the present consideration.

We use the van der Waals equation of state setting the different parameters of simulation at different $\varepsilon$ to get the local equality of the experimental and model «real» criteria of similarity. For example, when $\varepsilon=$ $=0.03$, the experimental values are $\mathrm{Ra}_{r}=1.27 \cdot 10^{4}$ and $\operatorname{Pr}_{r}=12.4$. As following from (9), these magnitudes correspond to $\mathrm{Ra}=45.4, \mathrm{Pr}=0.986$ which should be entered into the governing equations. As shown in Fig. 2, the experimental and model criteria of similarity are equal to each other locally at $\varepsilon=0.03$. Investigating near-critical convection near the other temperature distance from the critical point one should estimate parameters Ra and Pr again to be used in the governing equations. Though the numbers $\mathrm{Ra}$ and Pr do not include the physical properties of helium far from the critical point, they ensure the correspondence of convective process in physical and model near-critical fluids in the neighborhood of the certain temperature.

\section{Simulation of near-critical Rayleigh-Benard convection}

On the basis of the mathematical model described above we performed the 2D simulation of steady-state Rayleigh-Benard convection in helium along the critical isochore close to experiments in a flat Rayleigh-Benard cell (height $-1 \mathrm{~mm}$, diameter $57 \mathrm{~mm}$ ) [8,9]. The temperature of the top plate was kept at a fixed value, the bottom plate was heated very slowly ensuring a quasi-steady state at every instant.

We simulated a part of the whole cell (a square with a side $1 \mathrm{~mm}$ ) containing only a single roll. Conditions at vertical boundaries are assumed to be non-viscous and adiabatic, at horizontal boundaries they are viscous and isothermal. The temperature of the upper surface is $0.33 \mathrm{~K}$ above critical, the tempera-
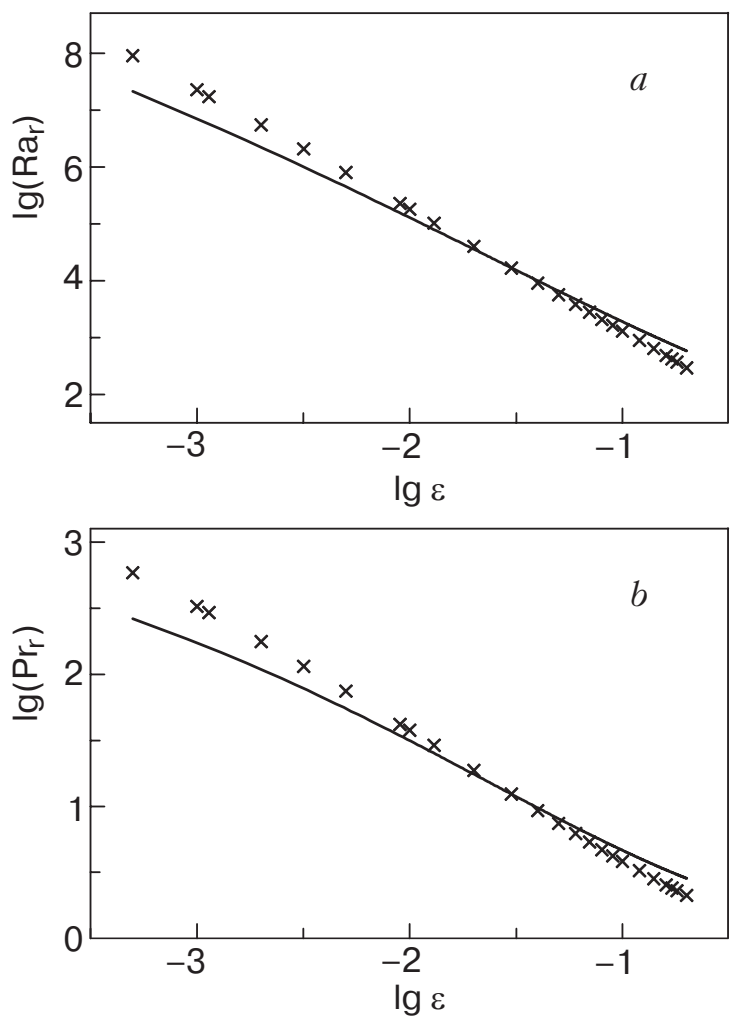

Fig. 2. The «real» Rayleigh $\operatorname{Ra}_{r}(a)$ and Prandtl $\operatorname{Pr}_{r}(b)$ numbers in the model fluid with local similarity to ${ }^{3} \mathrm{He}$ (solid lines) and in ${ }^{3} \mathrm{He}$ (marks) versus $\varepsilon$. 


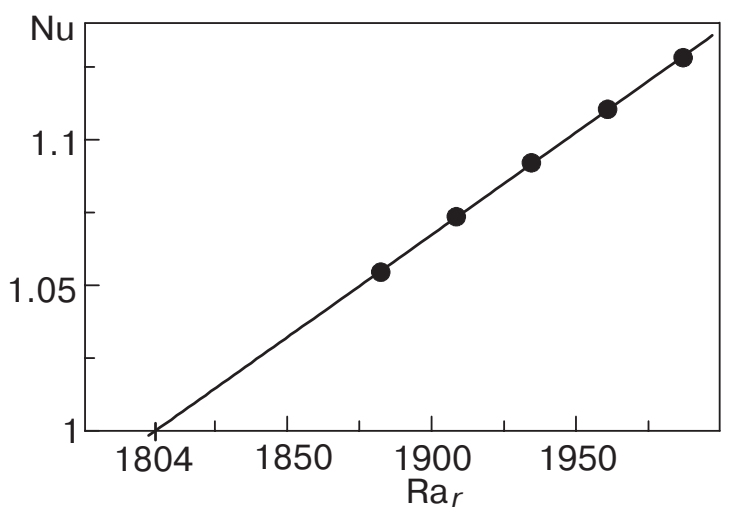

Fig. 3. The Nusselt number Nu versus the «real» Rayleigh number $\operatorname{Ra}_{r}$ (marks) and fitting linear curve (solid line) near the convection onset.

ture of lower surface is larger by some magnitude $\Theta^{\prime}$. The parameters $\varepsilon=0.1, \operatorname{Re}=8.33 \cdot 10^{3}, \operatorname{Pr}=0.814$, $\gamma_{0}=1.67, \mathrm{M}=10^{-3}$ are used. The temperature difference $\Theta$ and, consequently, the Rayleigh number Ra vary. We took $\Theta=(5.79-6.11) \cdot 10^{-6}$ (corresponding to dimensional values $\Theta^{\prime}=19.2-20.7 \mu \mathrm{K}$ and $\mathrm{Ra}=$ $=45.0-47.6$, respectively. The magnitudes of $\mathrm{Ra}$ and Pr are those to yield the «real» criteria of similarity $\mathrm{Ra}_{r}$ and $\operatorname{Pr}_{r}$ as in experiments.

The integration of the governing equations is started at the smallest value of $\Theta$ and it lasts until the steady-state regime is reached. Then the lower surface is slowly heated to the next value of $\Theta$ and integration is continued up to another steady-state regime. As a result, we have a set of stationary solutions at different $\Theta$. On the basis of these solutions one can receive the Rayleigh number $\mathrm{Ra}_{r \text {-onset }}$ characterizing the convection onset. We extrapolated the Nusselt number $\mathrm{Nu}$ to the value $\mathrm{Nu}=1$ where convection is absent. The $\mathrm{Ra}_{r}$ dependence of $\mathrm{Nu}$ is supposed to be a linear form when a fluid starts convecting. The extrapolation gives the threshold value $\mathrm{Ra}_{r \text {-onset }}=1804$. The relationship between $\mathrm{Nu}$ and $\mathrm{Ra}_{r}$ is shown in Fig. 3 .

The results obtained here are in a good agreement with experimental $[8,9]$ and theoretical [14] data concerning stability problems in compressible media. As predicted there the obtained value of $\mathrm{Ra}_{r \text {-onset }}$ is slightly larger then the classical magnitude in incompressible fluids (equals to 1708) because of the nonzero adiabatic temperature gradient. These results show that the general approach to simulation of near-critical dynamics developed above ensure the excellent correlation between heat transfer in model and real fluids under conditions of experiment.

\section{Discussion and conclusions}

The mathematical model for simulating near-critical dynamics, fitting to the known experimental data on helium, the results of simulation of gravity-driven convection and the approach to realizing obtained results were presented. Numerical simulation was performed using the novel 2D-numerical code on the basis of the full Navier-Stokes equations with two-scale splitting of the pressure and the van der Waals equation of state. We used the calibration laws to estimate the «real» criteria of similarity based on the physical properties of near-critical fluids. It was shown that in order to simulate the conditions of experiments more closely, one should agree the «real» Rayleigh and Prandtl numbers in model and physical media.

Simulation of steady-state Rayleigh - Benard convection in helium near the critical isochore, investigated early experimentally, was performed. The Rayleigh number characterizing the convection onset was defined from obtained numerical data. This number is shown to be in a good agreement with known experimental and theoretical values.

However, the «real» Rayleigh and Prandtl numbers, as known, describe dynamics and heat transfer completely only in the case of incompressible fluid (in Oberbeck-Boussinesque model). The analysis of simulated near-critical convection in common case is supposed to require further development taking into account the compressibility of medium. It was revealed [14] that in a near-critical fluid the influence of compressibility and adiabatic temperature gradient in Rayleigh-Benard convection enhances with tending to the critical point and leads to dominating role of the Schwarzchild criterion. The expression of the adiabatic temperature gradient for the van der Waals gas has done in [15]. Notice that we determined here the convection onset only at one sufficiently large reduced temperature $\varepsilon=0.1$. Experiments [9] have shown, that the role of the Schwarzchild criterion is not significant at this distance from the critical point. That is why no parameter of compressibility is involved into the calibration laws and the Schwarzchild number is not considered, but it should be taken into account for analysis of the closest near-critical neighborhood.

But nevertheless, the applied mathematical model, effective numerical code, and developed approach to realizing obtained results provide good possibilities to simulate and analyse the complex dynamic problems in near-critical fluids close to experimental conditions. This way allows to investigate numerically some characteristics of near-critical dynamics which are faced with difficulties in experiments. For example, the «piston effect» revealed in enclosures with a source of heating [16] is known to be very important in unsteady near-critical heat transfer. This effect may change the thermal and dynamic patterns sufficiently, 
but its influence is not always controlled experimentally. This problem may be solved numerically leading to well-studied and controlled conditions of experiments and understood results. All discussed points will be made out further.

\section{Acknowledgments}

The authors gratefully acknowledge Prof. H. Meyer for providing them with the experimental data on the physical properties of ${ }^{3} \mathrm{He}$ and for the careful correspondence. We also would like to thank Dr. A. Lednev for fruitful discussions. The work received financial support from the Russian Foundation for Basic Research (grant 03-01-00682).

1. H.E. Stanley, Introduction to Phase Transition and Critical Phenomena, Oxford Science Publications, New York (1987).

2. M.A. Anisimov, Critical Phenomena in Liquids and Liquid Crystals, Philadelphia, Gordon and Breach (1991).

3. V.I. Polezhaev and M.P. Vlasuk, Reports of the Russian Academy of Sciences. Hydromechanics 195, 1058 (1970) (Russian).

4. S. Amiroudine, P. Bontoux, P. Laroude, B. Gilly, and B. Zappoli, J. Fluid Mech. 442, 119 (2001).

5. A.G. Churbanov, A.N. Pavlov, A.V. Voronkov, and A.A. Ionkin, in: Proceeding of the Tenth International
Conference on Numerical Methods in Laminar and Turbulent Flow, Swansea, 1997, Pineridge Press, Swansea (1997), p. 1099.

6. V.I. Polezhaev and E.B. Soboleva, Izv. Akad. Nauk, Mekh. Zhidk. Gasa 1, 81 (2002) [Fluid Dynamics 37(1), 72 (2002)].

7. V.I. Polezhaev and E.B. Soboleva, Izv. Akad. Nauk, Mekh. Zhidk. Gasa 3, 143 (2001) [Fluid Dynamics 36 (3), 467 (2001)].

8. A.B. Kogan, D. Murphy, and H. Meyer, Phys. Rev. Lett. 82, 4635 (1999).

9. A.B. Kogan and H. Meyer, Phys. Rev. E63, 056310 (2001).

10. H. Meyer, Private communication.

11. E.B. Soboleva, Teplofiz. Vys. Temp. 38, 928 (2000) [High Temp. 38, 893 (2000)].

12. P. Schofield, Phys. Rev. Lett. 22, 606 (1969).

13. A.Z. Patashinskii and V.L. Pokrovskii, Fluctuation Theory of Phase Transition, Pergamon, New York (1979).

14. M. Gitterman and V.A. Steinberg, Teplofiz. Vys. Temp. 10, 565 (1972) [High Temp. 10, 501 (1972)].

15. V.I. Polezhaev, A.A. Gorbunov, and E.B. Soboleva, in: Proceeding of the Second Pan-Pacific Basin Workshop on Microgravity Sciences, Pasadena, 2001 [Adv. Space Res. 29, 581 (2002)].

16. A. Onuki, H. Hao, and R.A. Ferrell, Phys. Rev. A41, 2256 (1990). 